\title{
Materials for a Cercospora-Flora of the Kanto District. (1)
}

\author{
By \\ Shigetaka KATSUKI *
}

\section{Introduction}

The species of Cercospora occurring in the Kanto district have been described by P. HiinNINGS, P. \& H. Symow, N. Nambu, T. Yoshinaga, etc.. In their publications, more than 20 species including 11 new were reported. The new species are as follows:-

Cercospora Falouae P. HENN., C. Litieae P. Henn., C. Hibisci-Manilotis P. Henn., C. Araliae P. Inenn., C. P'unicae P. IIsnn., C., Miyakei P. Henn., C. Grassicicola P. ILEN., C. Vilisheie-ophyllae P. Hinn., C. Achyranthis SrD., C. oblegens SYD., C. Ze.kozua HIIRI.

The writer is intending to reconsider the species of Ce cospora in this district, standing on the current acceptance of classification of the genus. In this first report, 16 species are enumerated, of which C. Togashiana KATSUKI et URASAWA is ascertained to be new to science.

The writer wishes to express his hearty thanks to Dr. K. TugASHI, Yokohama National University and Dr. H. YoshII, Kyushu University for their valuable suggestions and kind guidance, and also Mr. Yoshihisa URASAwA, Iwatsuki Industrial. Senior High School, Saitama Pref,, for his kind help in collection of the materials examined.

(1) Cercospora Achyranthis Svioow Ann. Mycol. 7:171, 1909; SAcc., Syll. Fung. 22:1429, 1931; Solmeim and Stevens, Mycologia $23 ; 378$, ;231; TAI, Bull. Chinese Bot. Soc. 2 (2):45, 1936 ; K^Tsukı, Bull. Agric. Improvem. Sect. Econ. Dept. Fukuoka 1:3, 1949.

On Achyranehes japonica (Intokozuchi). I watsukimachi, Saitama Pref. (June 17, 1950, S. KATSUKI).

(2) Cercogpora Apii FrLSeniUs Beitr. Myk. 3:91, 1863; ELLIS et EVERHART, Jour. Myc. 1:36, 1885; Sacc., Syll. Fung. 4:442, 1886; S^wADA, Cat. Formosan Fung. 2: 140, 1923; Solheis, Illinoise Bio. Monog. 12(1):34-35, 1929; ChUp' \& DoIdGe, Bothalia 4(4): 881,1948 ; THIR UMALACHAR \& Chupp, Mycol. 40:353, 1948.

Syn. Cercospora penicillala var. Apii FLKL.,

- Agr. Inprov. Sect. Fukuoka pref, Govnt.
Hedwigia $2: 132-136,1863$.

On Apizm graveolens (Oranda-mitsuba). Ageomachi, Saitama Pref. (Nov. 17, 1949, H. Sekini).

(3) Ce: cospora beticola SAcc. Nuovo Giorn. Bot. Ital. 8: 189, 1876; SACc., Syll. Fung. 4: 456, 1886; AтKINSON, Jour. Elisha Mitchell Scient. Soc. $8: 14,1891$; N. Nambu, Bot. Mag. Tokyo, 18: 1-3, 19C4; Cilupl \& DoInce, Bothalia 4(4): 882, 1948; KA'sUkI, Bull. Agric. Improv. Sect. Econ. Dept. Fukuoka 1:5, 1949.

Syn. Cercospora flage!liformis EL1.. et HALSTED, N. Jersy Ann. Rept. for 1890, P. 355, 1891.

Cercospora Spitaciae OUD,-Nederl. Kruidk. Aruh. $3(2): 314,10 c 0 . \quad$ Cercosporina spinacicola SAcc., Nuov. Giorn. Bot. Ital, N. S. 22: 73, 1915.

On Bela vulgaris var. Rapa (Sato-daikon). Iwatsukimachi, Sailama Pref. (June 17, 1950, S. KATSUKI).

(4) Cercospora Bolleana (TuÜi.) SPEGAzIIA Mich. 1:475, 1879; Atrinson, Jour. Elisha Mitchell Scient. Soc. 8:29-30, 1891; SolHeim. Illinois Biol. Monog. 12(1):41, 1929; MURATA, Jour. Plant Prot. 2: 149, 1915.

On Ficus Caricu (Ichiziku). Iwatsuki-machi, Saitama Pref. (July 11, 1950, H. Skine).

(5) Cercospora carolae (PASSERINI) SOLHEIM Illinois Biolog. Monog. 12(1):43-44, 1929.

Syn. Cerco:pora apii FRES. var. carolae PASS. Mem. R. Acad. Linnei, Roma, $4(6): 469,1890$; SncC., Syll. Fung. 10:624, 1892; v. HöHNEI, Ann. Myc. 1: 530, 19('3; LINDAU, Rabenhorst's Kryptoga-

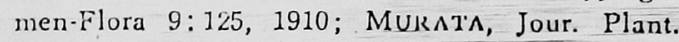
Prot. 8:185-186, 8:233-237, 1921. Cercospora

- carolae (PASS.) KAzNOWSKII \& SIEMASZKo, Centralbl. für. Bakt., Ab. 2, LXXVIII, 1-7, pp. 113-116, 1929 ; Rev. Appl. Myc. 8:630, 1929.

On Darcus Carola var. saliva (Ninjin). I wa. tsuki-machi, Saitama Pref. (June 17, 1950, S. KATSUKL).

The fungus occurring on carrot has hitherto been identified to C. apii FRLS. var. carolae PASS. in Japanese literature, but the writer is of opinion that $C$. carolac (P^SS.) SoLmlins is correct. The fungus under consideration is distinctly different 
from the several forms of C. apii FRES., in having by the former with light yellowish-brown and continuous conidiophores and with cylindrical or obclavate conidia.

( (i) Cercospora Chenopodii Fr. Beitr. Myk. 3: 92, 1863; Togasashi, Jap. Jour. Bot. 2(2):99, 1924; TAI, Bull. Chinese Bot. Soc. 2(2):49, 1936.

Syn. Cercospora dubia (RISS.) WINT. Hedwigia 22: 10, 1883; SYdow, Ann. Myc. 7: 171; 1969; KATSUki, Bull. Agric. Improv. Sect. Econ. Dept. Fukuoka 1: 12, 1949.

On Chenopodium album var. cenlroruburum (AkazA). Iwatsukimachi, Saitama Pref. (June 12, 1950, Y. URASAWA).

( 7) Cercospora Chloranthi TUGaSHI et KATSUkI Trans. Sapporo Nat. Hist. Soc. 17: \6-97, 1942; Katsuki, Bull. Agric. Improv. Sect. Econ. Dept Fukuoka 1: 83, 1949.

On Chloranthus serratus (Futari-shizuka). Iwatsukimachi, Saitama. Pref. (July 22, 1950, Y. URASAWA).

(8) Cercospoza Dispori Tigashi et MAkI Trans. Sapporo Nat. Hist. Soc. 17:98-99, 1942; KATSuri, Bull. Agric. Improv. Sect. Econ. Dept. Fukuolia 1: 12, 1949.

On Disporum sessile (Hochakuso). Iwatsukimachi, Saitama Pref. (July 20, 1950, Y. URAsawa).

This species was first described in 1942 by Dr. TUGASHl, and Disporum sessile is a new host plant to this fungus.

(9) Cercospora Fatouae P. Henings Fng. Bot. Jahrb. 32: 45, 1962; Hedwigia 43:146, 1904 ; Sacc., Syll. Fung. 18:610, 1ec6; N. NambU, Bot. Mag. Tokyo 18:3, 1904; Katsuki, Bull. Agric. Improv. Sect. Econ. Dept. Fukuoka 1: 14, 1949.

On Fatoua juponica (Kuwakusa). Niiwa-mura, Saitama Pref. (Sept. 22, 1949, Y. URASAWA).

- This species was first described in 1902 by P. HeNings, basing on the material collected by N. NAMUU in the vicinity of Tolkyo.

(10) Cercospora Nandinae Naga Tomo Kyoikushiryo (Materials for Education, Kyoto Pref.) No. 3, 1932; - Forsch. Geb. Pflanzenkrank. 3:110 114, 1837; K^TsUKI, Bull. Agric. Imoprov. Sect. Econ. Dept. Fukuoka 1:17, 1949.

Syn. Cercospora Nandinae FukuI, Bull. Imp. Coll. Agric. and Forest. Mie 3: 14-15, 1933.

On Nandiana doniestica (Nanten). I watsulkimachi, Saitama Pref. (June 17, 1950, S. KA'l'suki).

(11) Ceicospo:a Paederiae TaI Bull. Chinese Bot. Sэc. 2(2):56, 1936; K^tsuki, Bull. Agric. Improv. Sect. Econ. Dept. Fukuoka 1: 17, 1949.

On Paederia C'hinesis (Heliuso-kazura). I watsukimachi, Saitama Pref. (June 17, 1949, S. KATSUKI).
(12) Cercospora Hydrocotyles ELL. et Ev. Jour. Myc. 3: 16, 1887; SAcc., Syll. Fung. 10:624, 1892; AtKInson, Jour. Elisha Mitch. Scient. Soc. 8:23, 1892.

On Hydrocot yle sibchorpioides (Chidome-gusa). Iwatsuki-machi, Saitama. Pref. (June 17, 1950, S. KATSUKI).

(13) Cercospo-a Togashiana KATSUKI et URASAWA sp. nov. Maculis idefinitis, supra nonnihil pallide flavidis, infra griseis dein sordidis; caespitulis hypophyllis, stroma fusis vel nigricantibus, $22-30 \mu$. diam.; conidiophoris hypophyllis raro epiphyllis, fasciculatis, griseo-bruneis, simplicibus vel ramulosis, rectis vel flexuosis, raro geniculatis, distincte multiseptatis, $6 C-150 \times 3.7-5 \mu$; conidiis cylindraceis vel obclavato-cylindraceis, ad apicem leniter acutis, raro rectis, 4-8-septatis, ad septa interdum constrictis, $55-92 \times 3.7-5 \mu$ hyalinis.

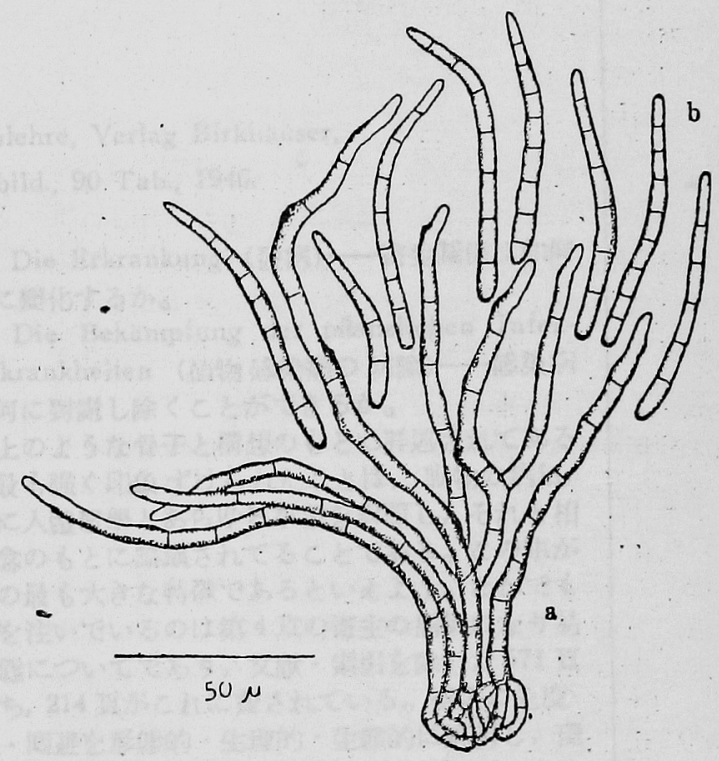

Fig. 1. Cercospora Togashiana KATSUKI et UR $\Lambda$ SA W' $A$ sp. nov.

a. Conidiophores b. Conidia

On Ophiopogon japonicus (Janohige). Niiwamura, Minamisaitama, Saitama Pref. (July 1, 1950, Y. URASAWA-Type !)

This species differs from Ce-cospora Liriopes TAI (Bull. Chinese Bot. Soc. 2: 55, 1936; Sci Rept. Nation. Tsing Hua Univ. Ser. B, 2: 431, 1937.) occurring on Liriofe graminifolia in having mostly hypophyllous, branched, longer, more septate conidiophores and hyaline conidia. The symptom is also different from that of C.Liriopes TAI. 
(14) Cercospora zebrina PAss. Hedwigia 16: 124, 1877; JА^P, Ann. Myc. 15: 124, 1917; S^cC., Syll. Fung. 4: 437, 1886; Horsiall, Mycologia 21: 304-312, 1929.

Syn. Cercospora helvola Sicc. Fungi Ital. pl. 667, 1881. C. Davissi ELLIS et EvEKLI. Proc. Acad. Nat. Sci. Phila. 43:89,1891, C. Medicaginis Els. et Ev. Proc. Acad. Nat. Sci. Phila. 43:91, 1891. C. Sto'riana P. MAGs.'Flora Tirol 3:558, 1905. Cercosporina ZEBRINA (Pass.) M^'rsuUk^, Jour. Plant. Prot. 17: 1-5, 1930.

On Trifolium repens (Shirotsume-gusa). Iwatsuki-machi, Saitama Pref. (June 6, 1950, Y.
URASAWA).

(15) Cercospora Zelkowae HORI, NANisU, Jour. Plant Prot. 8: 492, 1921; Is 1 iSUkI, Bull. Agric. Improv. Sect. Econ. Dept. Fukuoka 1:26, 1949.

On Zelkova serrata (keyaki). Hodogaya, Kanagawa Pref. (June 19, 1950, S. KATsUkI).

(16) Cercospora zonata WinT. SACC., Syll. Fung. 4:437, 1886.

Syn. Cercospora Fabae Fautk. Snce., Syll. Fung. 10:621, 1892.

On Vicia Falla (Soramame). Iwatsuki-machi, Saitama Pref. (May 17, 1950, Y. URASAwA).

\section{植物成染照}

Ernst GüUMANN: Pflanzliche Infektionslehre, Verlag Birkhïuser,

Basel, Schweiz. 611 S., 311 Abbild., 90 Tab., 1946.

Dr. GüUnanN は，且つては Vergleichende Morphologie der Pilze (1928) を著わし，とれは

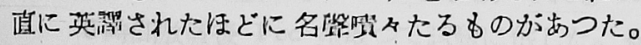
そればかりでなく, Peronosporaのモノグラフ, 土

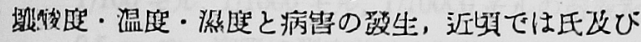
その共同矿究者との Antibiotik の矿独，さては森

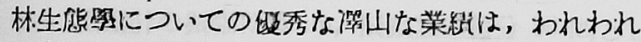
にも阿めて䢻染染い。最近は，また“Die Pilze”

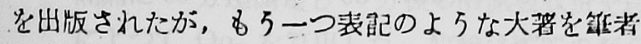
のもとに想啒して下さつた。

わが國でも，活行病照 (Epidemiology) なる当 装をよく耳にするようになつたが，氏の覞系ずけた

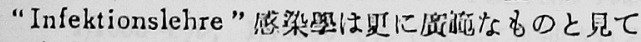

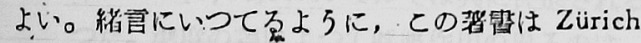
$の$ Eidgenössische Technische Hochschule にお。

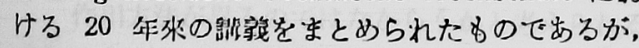

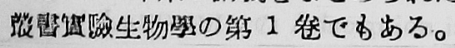

本翼は次の 6 棉からなつている。

1. Die Infektion（憾染）—病原は如何にして

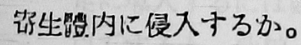

2. Die Infektketten (感染进颌) 一一疤原は如何

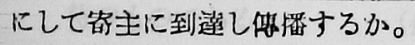

3. Die parasitische Eignung der Erreger (病

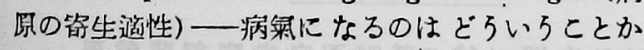
らか，病原の叫から見てどろか。

4. Die Krankheitsbereitschaft der Wir'e (宋)

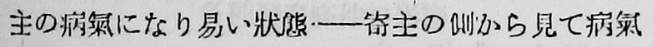
になるのは，どらいらことからぬ。

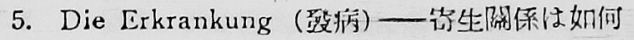
ように算化するか。

6. Die Bekämpfung der pflanzlichen Infektionskrankheiten (倠物柋染病の防除) 一一留染病 は如何に業䖍し除くことができるか。

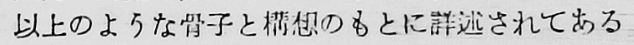

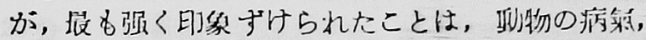

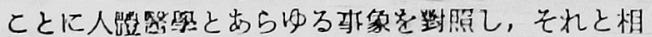

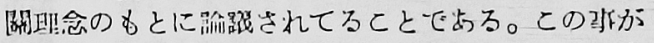

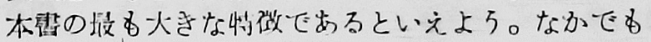

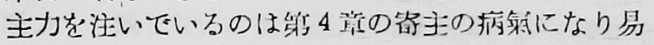

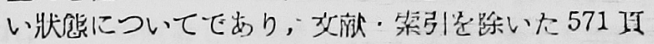
の5ち，214 頁かられに出されている。符主の化度。

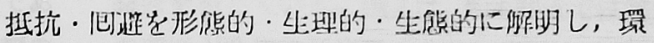
境要装の他に，Wuchsstoff, Anúibiotik, Lysine, Bakteriophage 灰大きく收入れてることる，仙の䑸 罾に見られない新しい睮成とみなせるであろら。

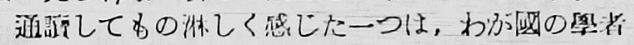

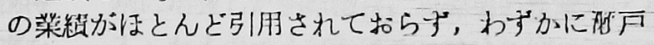

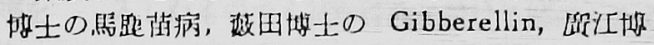
士の突然算買に路するもの位である。

ともあれ，Dr. Gn̈UNANN は值物病理學界の最高

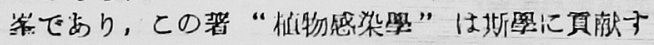
るとと赽炕大なるものあると信ずるし，フメリカ科

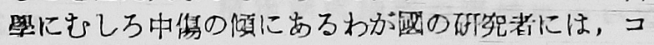
カコラの如くならぬ，新しい涌涼就となることを疑

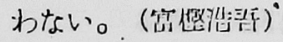

